\title{
Management of oral pemphigus vulgaris: A case report and a clinical update
}

European Journal of Inflammation 2015, Vol. I3(I) 53-57 (C) The Author(s) 2015

Reprints and permissions: sagepub.co.uk/journalsPermissions.nav DOI: $10.1177 / 1721727 \times 15576854$ eji.sagepub.com

(SAGE

\author{
F Pettini, ${ }^{* *}$ A Ballini, ${ }^{2 *}$ S Capodiferro, ${ }^{2 *}$ S Cantore, ${ }^{2}$ N Cirulli, ${ }^{2 *}$ \\ A Garofalo, ${ }^{3 *}$ MF Coscia, ${ }^{* *}$ D De Vito ${ }^{4 *}$ and C Foti ${ }^{3 *}$
}

\begin{abstract}
Pemphigus vulgaris (PV) is an uncommon autoimmune intraepithelial blistering disease. In most cases, the oral lesions were the first manifestation of the pathology. We report the case of a 42-year-old woman with a 4-month history of oral ulcerations. The patient reported that the lesions caused considerable discomfort and affected her normal oral function. On intraoral examination, ulcers were observed on the cheek and palatal mucosa and ventral surface of the tongue. No skin lesions were seen on extra oral examination. A diagnosis of PV was made after evaluating the biopsy samples. The main complication of PV is the reduced quality of life related to soreness or pain, particularly in ulcerative/erosive lesions. The presence of lesions among gingival tissues makes oral hygiene procedures very difficult, but plaque control and rigorous oral hygiene are a fundamental requisite for the treatment of any oromucosal disease.
\end{abstract}

\section{Keywords}

autoimmune blistering diseases, desmoglein, desquamative gingivitis, direct and indirect immunofluorescence, pemphigus vulgaris

Received 19 December 2014; accepted: 4 February 2015

\section{Introduction}

Pemphigus encompasses a group of autoimmune blistering diseases of the skin and mucous membranes. $^{1-3}$ Included in this group is pemphigus vulgaris (PV), a bullous disease involving the skin and mucous membranes, which may be fatal if not treated with appropriate immunosuppressive agents. ${ }^{3-5}$ The detection of circulating antibodies against keratinocyte surfaces led to the understanding that PV was an autoimmune disease..$^{3,6}$ In fact, patients with PV produce IgG autoantibodies against the desmoglein 3 (the 'PV antigen'), a transmembrane glycoprotein which mediates cell adhesion. ${ }^{6}$ Although the exact mechanism is unclear, autoantibodies theoretically produce an allosteric change in the desmoglein, impairing its adhesive abilities, and increase active plasmin in the area, producing cell degradation and acantholysis. ${ }^{6,7}$ Complement may be actively involved in this process. ${ }^{7}$ Characteristically, PV lesions usually start affecting the oral mucosa, followed by the appearance of skin lesions months later. Most patients are initially misdiagnosed and improperly treated for many months or even years. Dental professionals must be sufficiently familiar with the clinical manifestations of PV to ensure early diagnosis and course of the disease. ${ }^{8}$ The purpose of the

\footnotetext{
'Department of Interdisciplinary Medicine, University of Bari “Aldo Moro", Bari, Italy

${ }^{2}$ School of Medicine, University of Bari “Aldo Moro”, Bari, Italy 3Department of Biomedical Science and Human Oncology, Section of Dermatology, University of Bari “Aldo Moro”, Bari, Italy

${ }^{4}$ Department of Basic Medical Science, Neurosciences and Sense Organs, Section of Microbiology, University of Bari “Aldo Moro”, Bari, Italy *Equal contributors

Corresponding author:

Andrea Ballini, School of Medicine, University of Bari “Aldo Moro”, Piazza Giulio Cesare I I, 70I24 Bari, Italy.

Email: andrea.ballini@me.com
} 


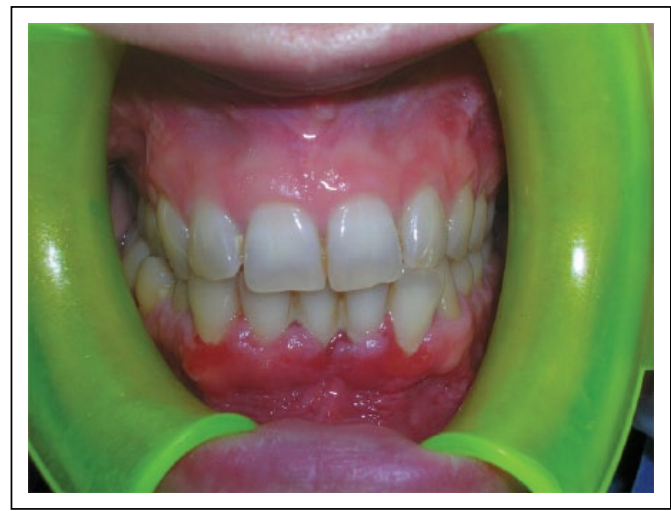

Figure I. Clinical presentation of oral lesions in pemphigus vulgaris: the gingivae.

current study was to present the management of a patient with an history of oral painful, who was finally diagnosed as having PV.

\section{Materials and methods}

\section{Clinical case}

We report a case of a 42 year-old woman referred to the Department of Dental Sciences and Surgery from the Dermatology Clinic for painful oral ulcers of 4 months' duration, leading to difficulties in chewing. Her medical history was significant only for multiple thyroids nodules. The intraoral examination revealed intense erythema of the gingiva (Figure 1); ulcers were visible on the cheek and palatal mucosa (Figure 2) and ventral surface of the tongue (Figure 3), and also on the oropharyngeal mucosa. No signs of cutaneous involvement were present except for an eye erosion.

Based on the history, multiple erosions and the apparent fragility of the gingivae experienced during examination, a vesiculobullous disorder was suspected. Pemphigus and pemphigoid were considered in the differential diagnosis. As mucous membrane pemphigoid is usually seen in the elderly, the former was thought to be more likely. Other common conditions that can present with similar manifestations are oral lichen planus or drug hypersensitivity. After obtaining written informed consent, a biopsy was drawn from perilesional site of the involved gingivae and the specimen was send for histological examination and direct and indirect fluorescence analysis.

During hospitalization the patient underwent various laboratory and instrumental investigations,

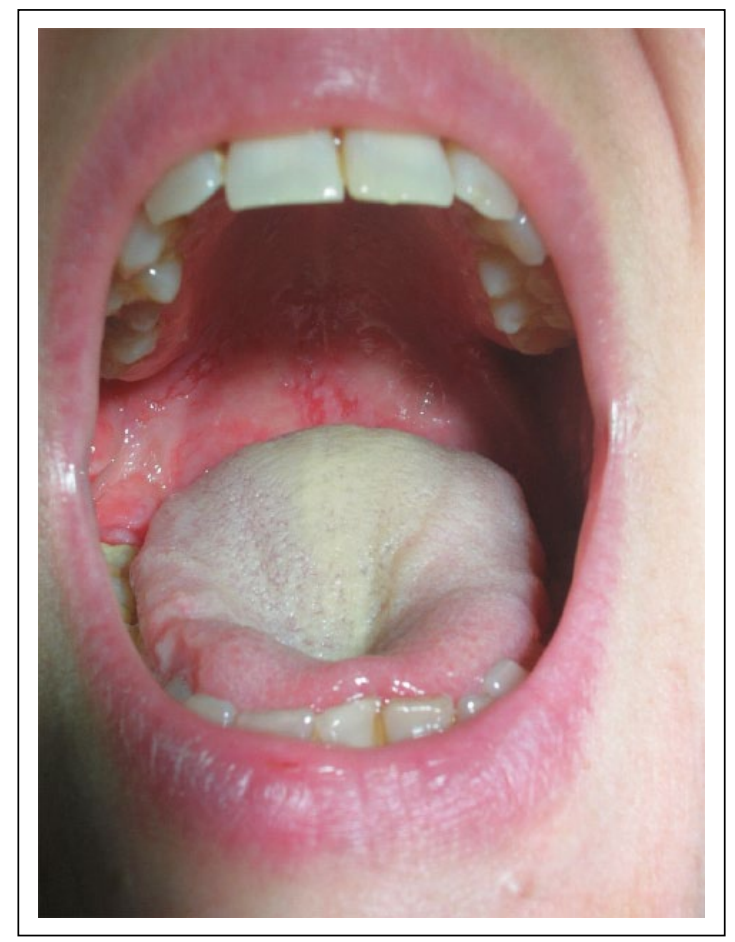

Figure 2. Clinical presentation of oral lesions in pemphigus vulgaris: the palatal mucosa.

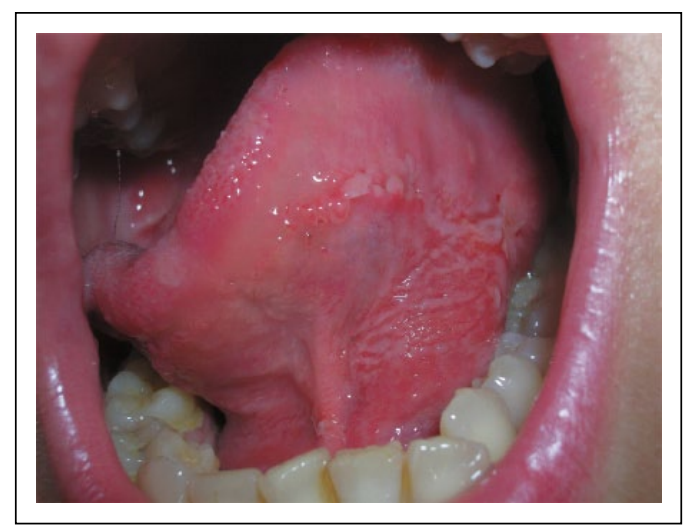

Figure 3. Clinical presentation of oral lesions in pemphigus vulgaris: the ventral surface of the tongue.

also performed consulting ophthalmologist, otorhinolaryngologist, and gastroenterologist.

Laboratory tests revealed normal value (n.v.) ranges except for: eosinophil (Eo) \%: 6.3 (n.v. = 0.00-4.00), total cholesterol: $208 \mathrm{mg} / \mathrm{dL}$ (n.v. $<200$ ), first hour erythrocyte sedimentation rate (ESR): $23 \mathrm{~mm} / \mathrm{h}$.

Urinalysis showed pH: 5.0 (n.v. $=5.5-6.5)$, protein: $20 \mathrm{mg} / \mathrm{dL}$ (n.v. $=0.00-10.00)$, ketones: 20 $\mathrm{mg} / \mathrm{dL}$ (n.v. $=0.00-0.00)$, leukocytes: $25 \mathrm{leu} / \mu \mathrm{L}$ (n.v. $=0.00-0.00)$. 
Microscopic examination of the sediment showed calcium oxalate crystals, rare red blood cell, different cells of squamous epithelium, the presence unclassified of crystals, and rare leukocytes well preserved.

The markers for hepatitis (A, B, and C) were negative.

\section{Assessment of oral tissue biopsy and microscopic examination}

Oral tissue biopsies were taken from the periphery of the lesions. The tissue was fixed in formalin and embedded in paraffin. Sections were stained with hematoxylin and eosin (H\&E) and examined using light microscope (Nikon Eclipse e800).

To evaluate inflammation, the number of inflammatory cells (mononuclear and polymorphonuclear cells) in the superficial parts of the connective tissue adjacent to the tip of the epithelial rete ridges were counted in six random fields (one field $=250 \mu \mathrm{m}^{2}$ ) per section using an ocular grid and high power magnification $(40 \times)$. The inflammatory cells were counted three times per each field, and results were expressed as a mean per specimen (mean $\left.\pm \mathrm{SD} / 1500 \mu \mathrm{m}^{2}\right)$. The variation of degree of inflammation between specimens was evaluated.

Histopathological examination revealed a suprabasal blister formation associated with extensive acantolysis of keratinocytes suggestive of PV (Figure 4), which was confirmed by direct immunofluorescence demonstrated $\operatorname{IgG}$ deposits in the intracellular spaces and on the soprabasal epithelium (Figure 5). Indirect immunofluorescence was positive (the screening dilution was 1:100).

A diagnosis of $\mathrm{PV}$ was performed.

\section{Patient management and follow-up}

Since the patient only had isolated oral involvement, she was started on systemic corticosteroids (prednisolone $1 \mathrm{mg} / \mathrm{kg} /$ day). This initial dose was stepped up to $2 \mathrm{mg} / \mathrm{kg} /$ day, which gave marked improvement in 2 weeks.

Oral treatment comprised an intensive individual hygiene program to establish the most appropriate non-traumatic procedures and to obtain the best possible standard of oral hygiene. At the beginning of the treatment, the patient was advised against the use of alcohol-based mouthwashes to avoid caustic damage to the damaged buccal mucosa.

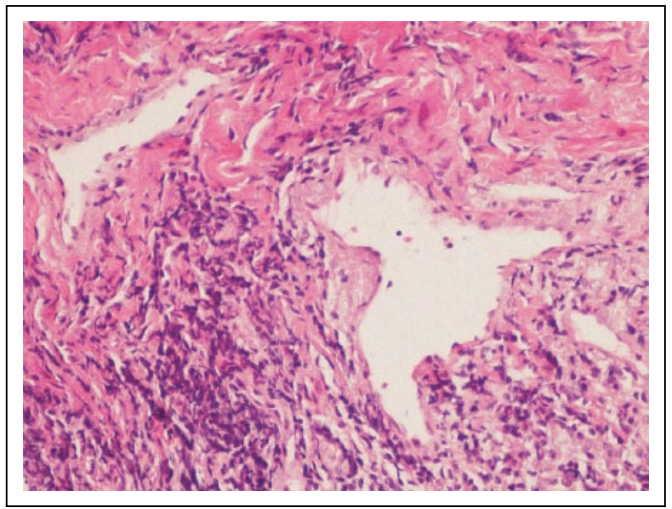

Figure 4. Histopathologic examination of specimens from the oral sample of pemphigus vulgaris shows acantholysis in the lower spinous cell layers. Basal layer cells are attached to the connective tissue and suprabasal cleft are seen at the tips of the epithelial rete ridges (scale $=200 \mu \mathrm{m})$.

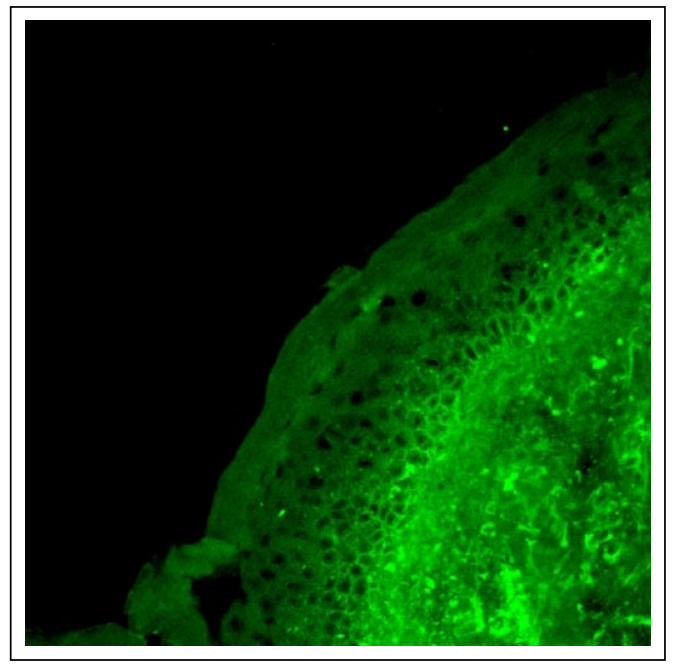

Figure 5. Direct immunofluorescent staining (immunoglobulin G) revealing pemphigus vulgaris antigen distribution in the oral epithelium. Note the 'spider-web' distribution of reaction product in the spaces between squamous epithelial cells (obtained from perilesional biopsy specimen containing intact epithelium).

A 'colorimetric' brushing technique was also recommended, using a liquid plaque detector, to identify the areas of greatest risk in the mouth and to offer an effective plaque control.

The patient was instructed to apply twice daily topical steroid oral paste (triamcinolone acetonide $0.1 \%$ ) along with local anesthetic gel. The patient was instructed to use a soft brush for brushing the teeth. In addition, an antifungal mouthwash was given to prevent secondary fungal infection. Sometime after commencement of corticosteroid therapy, scaling and root planing were performed. 
The patient was placed on a diet with no spicy food or alcohol, and was also registered with an anti-smoking counseling program. The patient underwent regular check-ups to control both the disease and her oral hygiene every month for the first 3 months, and subsequently every 6 months.

Over the past 1 year, the prednisolone has been tapered to $10 \mathrm{mg} /$ day. Currently, the patient is on this daily low-dose systemic corticosteroid therapy (prednisolone), topical steroid oral paste (triamcinolone acetonide 0.1\%), and supplementary medications.

In addition, to date, all last laboratory tests revealed normal value ranges, and no other sites are involved.

\section{Discussion}

For Scully et al. ${ }^{9}$ the oral mucosa is almost always affected in patients with $\mathrm{PV}$, and in $50-70 \%$ of cases it is the first site to be involved. The authors add that such manifestations are the most resistant to the applied therapeutics, anticipating cutaneous lesions in months or even years. ${ }^{9}$

The mouth is the only site of involvement in half of all cases of PV, and is the initial site of presentation. Blisters can be created by pressure or friction upon a normal-appearing area of mucosa or skin (Nikolsky sign). A history of trauma-induced bullae and a positive Nikolsky's sign can help for the differential diagnosis, but a biopsy containing intact epithelium and direct immunofluorescence (DIF) are required for a definitive diagnosis. ${ }^{3,10,11}$ Histopathology should reveal suprabasal acantholysis, instead DIF, using the patient's perilesional epithelium as substrate, shows IgG deposition in the intercellular spaces between the keratinocytes. Desmoglein 3, a $130 \mathrm{Kda}$ component of desmosome, is the target antigen in this disease. ${ }^{6,7}$ The most important aspect of $\mathrm{PV}$ is its early recognition, diagnosis, and treatment. ${ }^{11}$ Dermatological examination is mandatory for all PV patients to evaluate the presence of skin or mucous lesions.

In this article we describe the management of a case of PV. We also distinguish the diagnosis of oral PV, which often results in the patient's death, if untreated, from other similar lesions and the importance of the roles of dentists in early diagnosis and treatment. ${ }^{8}$
In the present case study, the oral lesions were the first manifestation of the disease. However, the patient's oral hygiene was very poor and this in turn contributed further to gingival inflammation, leading to generalized periodontitis.

The initial aim of treatment is to induce disease remission. Corticosteroids are the mainstay of treatment for patients with PV. Prednisolone $(1 \mathrm{mg} / \mathrm{kg} /$ day), with or without other immunosuppressive agents, should be initiated immediately. ${ }^{12,13}$ It should be continued until there is cessation of new bullae formation and Nikolsky's sign can no longer be elicited. The dosage is then reduced by one half until all of the lesions have cleared, followed by tapering to a minimum effective maintenance dosage.

Much of the recent research has been assessing the efficacy of steroid-sparing agents, most commonly azathioprine, mycophenolate mofetil, methotrexate, intravenous immunoglobulin, cyclophosphamide, and Rituximab, an anti-CD20 monoclonal antibody. ${ }^{11}$ In severe cases, plasmapheresis may be required. ${ }^{5}$

Desquamative gingivitis causes severe pain and interfering with appropriate oral hygiene, leading to plaque accumulation. , $^{9} 14$

It is essential that dentists know these pathologies to be able to diagnose them in an early stage of the disease and to direct patients to adequate treatment. ${ }^{15}$ Furthermore, intraoral examination should be included as a routine practice in dermatological services. ${ }^{16,17}$

In the present case, according to literature data, ${ }^{18}$ systemic and topical corticosteroid treatment and adjuvant therapy of oral hygiene, antifungal mouthwash, use of soft brush, and vitamin supplementation were instituted.

In conclusion, in patients with PV who have lesions confined to the oral cavity, close follow-up is essential, and referral to specialists should be undertaken promptly in the event of appearance of extraoral symptoms.

The management of the patient suffering from desquamative gingivitis such as PV requires the synergic treatment of both dentist and dental hygienist, whose contribution supports the corticosteroid and/or immunosuppressive treatment, provided from the dermatologist.

\section{Declaration of conflicting interests}

The authors declared no potential conflicts of interest with respect to the research, authorship, and/or publication of this article. 


\section{Funding}

This research received no specific grant from any funding agency in the public, commercial, or not-for-profit sectors.

\section{References}

1. Kershenovich R, Hodak E and Mimouni D (2014) Diagnosis and classification of pemphigus and bullous pemphigoid. Autoimmunity Reviews 13: 477-481.

2. Ingen-Housz-Oro $S$, Hüe $S$, Grootenboer-Mignot $S$ et al. (2013) Auto-immune bullous diseases autoantibodies (pemphigus, bullous pemphigoid): What the dermatologist must know. Annals de Dermatologie et Venereologie 140: 563-570.

3. Scully C and Challacombe SJ (2002) Pemphigus vulgaris: Update on etiopathogenesis, oral manifestations, and management. Critical Reviews in Oral Biology \& Medicine 13: 397-408.

4. Williams DM (1989) Vesiculobullous mucocutaneous disease: Pemphigus vulgaris. Journal of Oral Pathology \& Medicine 18: 544-553.

5. Stanley JR (1999) Pemphigus. In: Freedberg IM, Eisen AZ, Wolff K, et al. (eds) Fitzpatrick's Dermatology in General Medicine. 5th edn. New York: McGraw-Hill, pp.654-666.

6. Hertl M, Jedlickova H, Karpati S et al. (2015) Pemphigus. S2 Guideline for diagnosis and treatment - guided by the European Dermatology Forum (EDF) in cooperation with the European Academy of Dermatology and Venereology (EADV). Journal of the European Academy of Dermatology and Venereology 29: 405-414.

7. Spindler V and Waschke J (2014) Desmosomal cadherins and signaling: Lessons from autoimmune disease. Cell Communication and Adhesion 21: 77-84.
8. Cirillo N, Cozzani E, Carrozzo M et al. (2012) Urban legends: Pemphigus vulgaris. Oral Disease 18: 442-458.

9. Scully C, Paes de Almeida O, Porter SR et al. (1999) Pemphigus vulgaris: The manifestations and longterm management of 55 patients with oral lesions. British Journal of Dermatology 140: 84-89.

10. Ramirez-Amador VA, Esquivel-Pedraza L and Orozco-Topete R (2000) Frequency of oral conditions in a dermatology clinic. International Journal of Dermatology 39: 501-505.

11. Daniel BS and Murrell DF (2014) Management of pemphigus. F1000Prime Reports 6: 32.

12. Mourellou $\mathrm{O}$, Chaidemenos CG, Koussidou $\mathrm{T}$ et al. (1995) The treatment of pemphigus vulgaris: Experience with 48 patients seen over an 11-year period. British Journal of Dermatology 133: 83-87.

13. Pandya AG and Sontheimer RD (1992) Treatment of pemphigus vulgaris with pulse intravenous cyclophosphamide. Archives of Dermatology 128: 1626-1630.

14. Ballini A, Scattarella A, Crincoli V et al. (2010) Surgical treatment of gingival overgrowth with 10 years of follow-up. Head \& Face Medicine 6: 19.

15. Ballini A, Capodiferro S, Toia M et al. (2007) Evidence-based dentistry: What's new? International Journal of Medical Sciences 4: 174-8.

16. Goncalves LM, Bezerra Junior JRS and Cruz MCFN (2010) Clinical evaluation of oral lesions associated with dermatologic diseases. Anais Brasileiros de Dermatologia 85: 150-156.

17. Hooten JN, Hall RP and Cardones AR (2014) Updates on the management of autoimmune blistering diseases. Skin Therapy Letter 19: 1-6.

18. Javali MA and Zainab H (2012) Pemphigus vulgaris presenting as gingival involvement. Indian Dermatology Online Journal 3: 202-204. 